Classification

Physics Abstracts

$61.50 \mathrm{~J}-63.90$

\title{
Fractal structure of the equilibrium crystal shape
}

\author{
S. E. Burkov \\ Landau Institute for Theoretical Physics, Moscow, U.S.S.R.
}

(Reçu le 20 juin 1984, accepté le 11 octobre 1984)

\begin{abstract}
Résumé. - Nous avons obtenu la forme d'équilibre d'un cristal classique à température nulle dans le cadre d'un modèle négligeant les distorsions atomiques. Dans ce modèle, l'interaction entre deux atomes d'une même paire décroît avec la distance plus rapidement que $r^{-4}$. Nous montrons que la surface du cristal a une structure fractale qui consiste en un nombre infini de facettes, d'arêtes et de sommets qui forme un ensemble de Cantor. La surface consiste uniquement en facettes (il n'y a pas de zone arrondie) mais la pente reste continue aux arêtes. Le bord d'une face a également une structure fractale. Elle consiste en un nombre infini d'arêtes " continues " et d'un ensemble de Cantor. Nous avons également obtenu la surface microscopique de chaque facette.
\end{abstract}

\begin{abstract}
The equilibrium crystal shape of the classical crystal at zero temperature is found in the framework of the model suppressing the atomic distortions. In this model the interaction between two atoms of a pair is supposed to decrease at large distances more rapidly than $r^{-4}$. The crystal surface is shown to have a fractal structure : it consists of an infinite number of facets and of solitary edges and corners, which form the Cantor set. The crystal is completely faceted (i.e. there is no rounded area), but there is no sharp edge (i.e. the slope is continuous). The boundary of a facet is shown to have a fractal structure too. It consists of an infinite number of " smooth " edges and of the Cantor set. The microscopic configuration of steps and kinks on every facet is also found.
\end{abstract}

Recently the equilibrium crystallization phenomena have become the object of attention of both experimentalists and theoreticians. This interest in equilibrium properties has been promoted by the experimental observation of the helium crystal faceting phenomenon [1, 2]. Prior to this experiment the equilibrium crystal shape (ECS) could not be observed due to a very large relaxation time. Solid ${ }^{4} \mathrm{He}$ is a quite rapidly relaxing substance. Quantum and thermal fluctuations in this substance play an important role. Nevertheless, it is interesting to investigate a crystal shape neglecting fluctuations of both types. Thermal fluctuations are minor at low temperatures whereas quantum fluctuations cannot be ignored. However the surface of a quantum crystal appears to differ from that of a classical crystal more quantitatively than qualitatively. More exactly, quantum fluctuations do not destroy facets on the surface $[3,4]$. For this reason many general zero temperature properties of the classical crystal can be observed in helium experiments.
Recently the observation of equilibrium faceting of small lead crystals has been reported [17]. The temperature of $10 \mu \mathrm{m}$ samples in the experiment is about $600 \mathrm{~K}$ and quantum fluctuations are negligible. Thermal fluctuations are important at $600 \mathrm{~K}$ and the observed ECS differs from the zero-temperature ECS. The latter can be measured at lower temperatures. We have exactly calculated the ECS of a model crystal at zero temperature. The model does not describe both solid ${ }^{4} \mathrm{He}$ and lead but we hope that some of the predicted phenomena are quite general and can be experimentally observed. We consider a " lattice gas" model with an infinite range of interaction. The crystal consists of identical atoms forming some simple lattice and interacting with each other. For the sake of simplicity we shall deal with a cubic lattice (the lattice constant is put equal to unity) although all the results are valid for an arbitrary simple lattice. The energy $U(\mathbf{r})$ of the interaction between every two particles is assumed to satisfy the conditions : 
1) $U(\mathbf{r}) \rightarrow 0$ as $|\overline{\mathbf{r}}| \rightarrow \infty$, namely $U(\mathbf{r})$ tends to zero more rapidly than $r^{-4}:|U(\overline{\mathbf{r}})|<c r^{-\gamma}, \gamma>4$. 2) $U(\overline{\mathbf{r}})<0$ at $|\overline{\mathbf{r}}| \geqslant 1$.

The typical function $U(\mathbf{r})$ corresponding to the repulsion at small distances and to the attraction at large distances is shown in figure 1 . Note that $U(\mathbf{r})$ is not necessarily only $|\overline{\mathbf{r}}|$ dependent.

The main feature of this model is that the lattice is supposed to be undeformable. Namely, the coordinates $(x, y, z)=\overline{\mathbf{r}}$ of the atoms must be integer : $\overline{\mathbf{r}} \in \mathbb{Z}^{3}$. Unfortunately, the elastic deformation near the surface of the real crystal can be important. Nevertheless, it is known that there are situations when atomic distortions are small. In these cases the lattice gas model adequately describes the real substance. In any case, most theoretical investigations have been performed in terms of this model. The role of elasticity will be discussed at the end of the paper.

It should be noted that the conditions 1 and 2 imposed on $U(\mathbf{r})$ are sufficient in the framework of the present "lattice gas" model. But it is clear that if the interatomic interaction $U(\mathbf{r})$ is rather exotic the model itself does not describe any physical situation. For example, $U(\mathbf{r})$ may not be monotonous at large $|\overline{\mathbf{r}}|$, i.e. the attraction is changed by the repulsion, etc. All the results described below are valid also in this case. But it is unlikely that particles with such an interaction form a simple cubic lattice. So, in this case a "lattice gas" model is a mathematical abstraction. A realistic potential $U(\mathbf{r})$ is shown in figure 1 . Most of the interaction energies in real substances are of this form.

So, we consider a system of $N$ atoms interacting with each other and situated at integer points. The energy is

$$
E_{N}=\frac{1}{2} \sum_{i, j=1}^{N} U\left(\overline{\mathbf{r}}_{i}-\overline{\mathbf{r}}_{j}\right) \quad \overline{\mathbf{r}}_{i}, \overline{\mathbf{r}}_{j} \in \mathbb{Z}^{3} .
$$

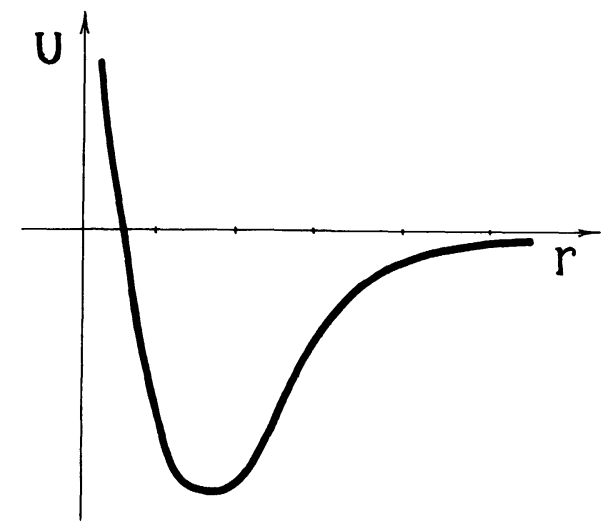

Fig. 1. - The energy of the interaction $U(\overline{\mathbf{r}})$ between two particles.
At large $N$ it can be presented as $E_{N}=\varepsilon N+E_{\mathrm{S}} N^{2 / 3}$, where $\varepsilon$ is an energy per particle, $E_{\mathrm{S}}$ is a surface energy. Since the temperature is zero and there are no quantum fluctuations the configuration of atoms providing an absolute minimum of $E_{N}$ must be found. Minimizing the leading term $\varepsilon N$ in $E_{N}$ gives the geometry of the lattice. We do not perform this minimization, supposing that $U(\bar{r})$ is such that the energy per particle $\varepsilon$ is minimal when atoms form a cubic lattice with a unit lattice constant. The energy $\varepsilon$ does not depend on the crystal shape, whereas the next term $E_{\mathrm{S}} N^{2 / 3}$ in $E_{N}$ is shape dependent.

So, the ECS is a configuration of atoms giving an absolute minimum of the surface energy $E_{\mathrm{s}}$, provided the particles form an undeformable cubic lattice. We assume that the volume of the crystal is fixed, which corresponds to the crystal-vacuum interface.

We are interested in the limit $N \rightarrow \infty$, and the problem of minimization is divided into two subproblems. To distinguish between them we must first consider the case of a large but finite $N$. In this case the ECS is given by a function $\bar{R}_{N}\left(\xi_{1}, \xi_{2}\right)$, where $\bar{R}_{N}$ is a radius-vector of the points on a crystal surface and $\xi_{1}, \xi_{2}$ are two real variables parameterizing the surface (e.g. $\xi_{1}, \xi_{2}$ may be two polar angles $\theta, \varphi$ ). The vector $\bar{R}_{N}$ has integer components : $\bar{R}_{N} \in \mathbb{Z}^{3}$. When we change the number of particles $N, \bar{R}_{N}$ increases as $N^{1 / 3}$. So, we must introduce a new radiusvector $\bar{R}\left(\xi_{1}, \xi_{2}\right)$ which does not depend on the crystal volume :

$$
\bar{R}\left(\xi_{1}, \xi_{2}\right)=\lim _{N \rightarrow \infty} \frac{\bar{R}_{N}\left(\xi_{1}, \xi_{2}\right)}{N^{1 / 3}}
$$

It is clear that $\bar{R}$ can take any real value, the steps of $\bar{R}_{N}$ are smoothed when we calculate the limit (2). So the $\operatorname{ECS} \bar{R}\left(\xi_{1}, \xi_{2}\right)$ may be a usual differentiable function. It is obvious, that since $\bar{R}$ does not depend on crystal volume it determines the shape of the sample (spherical, cubic, ellipsoidal, etc.) but not its size. It means that if $\bar{R}$ is a solution of the problem, then $\lambda \bar{R}$ is also a solution ( $\lambda$ is an arbitrary real number). Let this problem be called macroscopic.

It is well known that the crystal may be faceted. In the limits of the macroscopic approach a facet is described by a plane region of a finite size on the surface $\bar{R}\left(\xi_{1}, \xi_{2}\right)$. The function $\bar{R}\left(\xi_{1}, \xi_{2}\right)$ determines the shape of a facet and its area (more exactly, $\bar{R}\left(\xi_{1}, \xi_{2}\right)$ can give only the ratio of the facets' area to the total surface area). Facets are distinguished from each other by their Miller indices $\{p, q, r\}$ yielding the unit vector $\overline{\mathbf{n}}=(p, q, r) / \sqrt{p^{2}+q^{2}+r^{2}}$ normal to the facet.

We can fix some normal $\overline{\mathbf{n}}$ and consider the corresponding plane microscopically. Namely, we consider the function $\bar{R}_{N}\left(\xi_{1}, \xi_{2}\right)$ (with integer values) confining ourselves to a given facet. Its size tends to 
infinity when $N \rightarrow \infty$ because now $\bar{R}_{N}$ is not divided by $N^{1 / 3}$. Thus, the microscopic problem is to find the configuration of steps and kinks which gives an absolute minimum of the surface energy, provided the infinite crystal vacuum interface is a plane of a given orientation $\overline{\mathbf{n}}$. The second aspect of the microscopic problem is to calculate the surface tension $\alpha(\bar{n})$.

\section{Microscopic approach.}

Consider a plane $\overline{\mathbf{n}} \cdot \overline{\mathbf{r}}=0$ of some orientation $\overline{\mathbf{n}}$. There is an infinite number of configurations of steps and kinks having the same average slope (see Fig. 2). It is necessary to find among them the configuration minimizing the energy. In the limits of this model we formulate.

The fundamental assertion. The configuration in which the atoms occupy all the integer points $\overline{\mathbf{r}}$ lying below the plane $\overline{\mathbf{n}} \cdot \overline{\mathbf{r}}=0$, i.e.

$$
\overline{\mathbf{n}} \cdot \overline{\mathbf{r}} \leqslant 0, \quad \overline{\mathbf{r}} \in \mathbb{Z}^{3}
$$

provides an absolute energy minimum. In other words, any surface reconstruction is energetically unfavorable.

The assertion is not trivial. There are real crystals where the surface reconstruction takes place. In the model considered, this assertion is a theorem. The proof is given in Appendix 3. It has been obtained recently by $A$. Kerimov [5] and it is shorter than the original version. Note that the assertion is valid for any dimension of the lattice.

The atomic structure of the facet $\{2,3,8\}$ constructed using equation (3) is shown in figure $3 \mathrm{a}$. A projection of the 3-D picture on the $x y$-plane is shown in figure $3 b$. Every staircase-like line is the section of the interface by the parallel planes $z=0, z=1$, $z=2$, etc. Such a projection determines the structure of the facet as well as the 3-D picture (Fig. 3a). Note that the facet $\{p, q, r\}, p, q, r \in \mathbb{Z}$ has a periodic atomic structure. A surface cell is a parallelogram with the sides :

$$
\bar{A}_{1}=\left(T_{x},-T_{y}, 0\right) \quad \bar{A}_{2}=\left(-\left(T_{x}, 0, T_{z}\right)\right)
$$
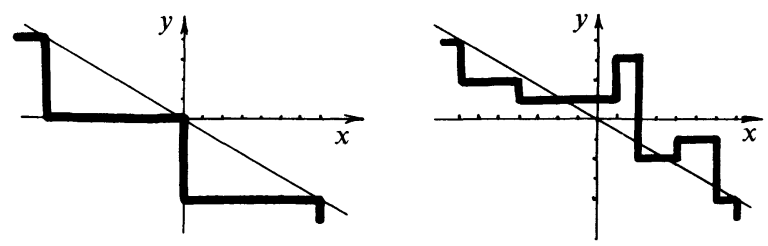

Fig. 2. - Two different configurations of steps with the same average slope $4: 7$.
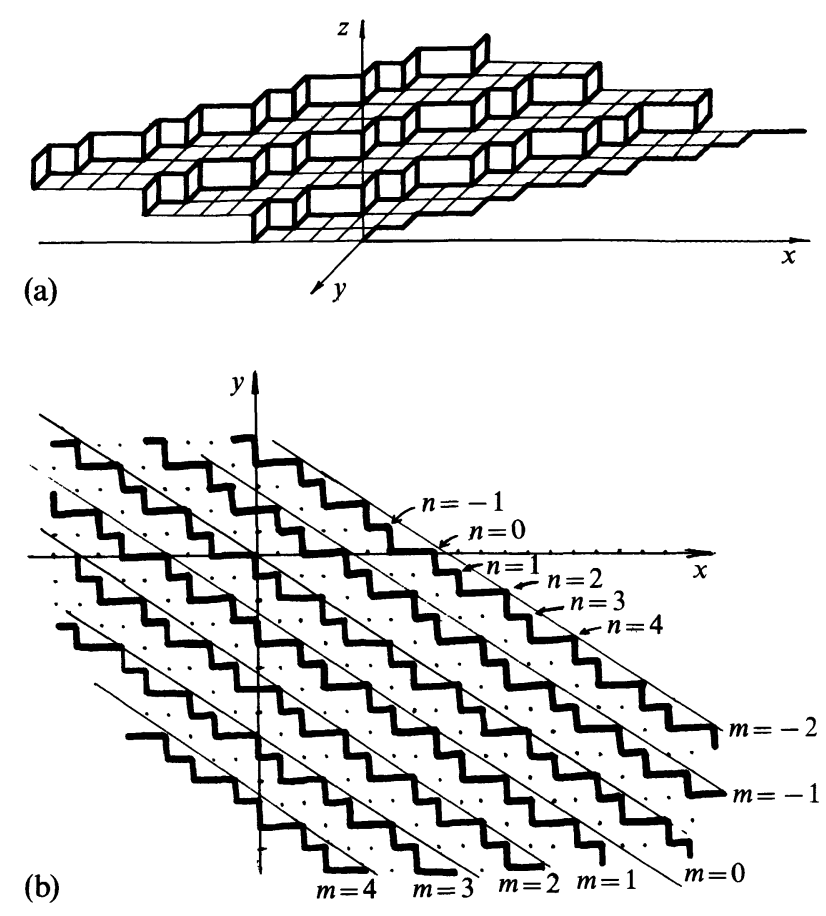

Fig. 3. - The configuration of steps and kinks on a facet $\{2,3,8\}$ constructed by means of the formula (8). A surface cell (a) is a parallelogram with the sides : $\bar{A}_{1}=(12,-8,0)$ and $\bar{A}_{2}=(-12,0,3)$ (see Eq. (4)). The slopes are $2 / 3$ in the $x y$-plane, $3 / 8$ in the $y z$-plane and $1 / 4$ in the $x z$-plane. (b) The projection on the $x y$-plane.

and with the diagonal $\bar{A}_{3}=\left(0,-T_{y}, T_{z}\right)$ where $T_{x}, T_{y}, T_{z}$ are :

$$
T_{x}=\frac{k}{p} \quad T_{y}=\frac{k}{q} \quad T_{z}=\frac{k}{r}
$$

and $k$ is the least common multiple of $p, q, r$.

Although the fundamental assertion determines the atomic structure of facets unambiguously, it is useful to obtain an analytical description. We start with the simplest case $\overline{\mathbf{n}}=\left(n_{x}, n_{y}, 0\right)$. There are no kinks on the steps and the picture is two-dimensional (Fig. 4). The steps are arranged according to the fundamental assertion. Their configuration $\left\{x_{n}, y_{n}\right\}$ can be described analytically :

For any facet $\{p, q, 0\}, p \leqslant q$ the coordinates of the steps are :

$$
x_{n}=\operatorname{Int}\left(n \frac{q}{p}\right), \quad y_{n}=-n, \quad n \in \mathbb{Z} .
$$

The formula $y_{n}=-n$ shows that the height of each step $y_{n+1}-y_{n}$ is unity. The spacing $x_{n+1}-x_{n}$ between the neighbouring steps can take only two values : Int $\left(\frac{q}{p}\right)$ or $\operatorname{Int}\left(\frac{q}{p}\right)+1$. Moreover, the spacing between the $n$-th and the $m$-th steps may be either Int $\left(|n-m| \frac{q}{p}\right)$ or Int $\left(|n-m| \frac{q}{p}\right)+1$. This 

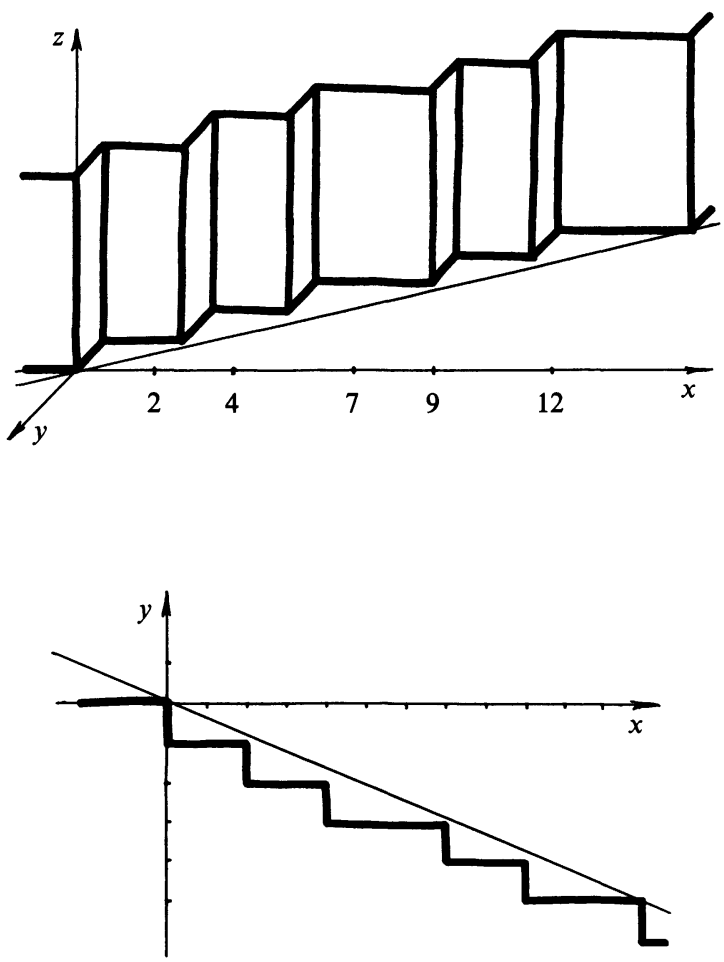

Fig. 4. - The configuration of steps on a facet $\{5,12,0\}$.

property can be easily derived from equation (5) (see e.g. [6]) and is called the Hubbard criterion [7]. The sequence of integers $\left\{x_{n}\right\}$ defined by equation (5) possesses some other interesting properties (see $[8,9])$.

This two-dimensional situation has been considered in $[10,11]$. The configuration of steps $\left\{x_{n}\right\}$ has been found for the simplest $p / q$, but the result has been presented in a more cumbersome form than equation (5). The analysis of $[10,11]$ has been based on the analogy between this model and 1-D lattice gas, a step playing the role of a particle. This analogy is exact (see Appendix 1) but the authors of $[10,11]$ have used approximate expressions for the energy of steps and the interaction between them. The fundamental assertion enables such a description to be avoided and yields equation (5) directly. Actually to derive equation (5) from equation (3) it is necessary to consider figure 4 bearing in mund that $n \frac{q}{p}-1 \leqslant$ Int $\left(n \frac{q}{p}\right) \leqslant n \frac{q}{p}$. Moreover, the fundamental assertion makes it possible to obtain analytical formulae for the 3-D situation.

Consider now an arbitrary facet with a normal $\overline{\mathbf{n}}=(p, q, r) / \sqrt{p^{2}+q^{2}+r^{2}}$. The plane is defined by the equation

$$
p x+q y+r z=0 .
$$

In the case $p \leqslant q \leqslant r$ it is convenient to consider the projection of the 3-D picture on the $x y$-plane (in the other case the coordinates $x, y, z$ should be permuted). At first we give a geometrical description of the configuration of steps and kinks (Figs. 3b, 5). We draw a system of parallel lines in the $x y$-plane :

$$
p x+q y=r m \quad m=0, \pm 1, \pm 2, \ldots
$$

The distances between them are $r / p$ in the $x$-direction and $r / q$ in the $y$-direction. The $m$-th line (7) is the intersection line of the two planes : $z=-m$ and $p x+q y+r z=0$. Then we draw staircase-like lines below each line (7) so that they are the nearest ones to the corresponding straight lines (Fig. 5). The obtained lines are the projections of steps on the $x y$ plane. This geometrical rule is only the fundamental assertion reformulated in terms of steps and kinks. Although the rule determines the configuration unambiguously we want to obtain analytical formulae. There are three equivalent methods. The first one is formulated as :

For any facet $\{p, q, r\}, p \leqslant q \leqslant r$ the coordinates

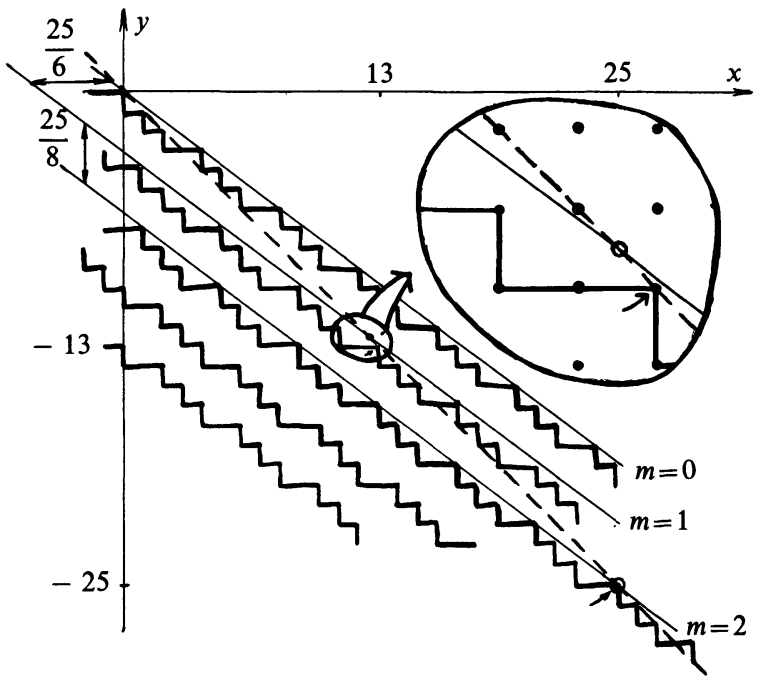

Fig. 5. - The projection on the $x y$-plane of the configuration of steps and kinks on a facet $\{6,8,25\}$. The lines $6 x+8 y=25 m$ (see Eq. (7)) are shown by solid lines. The fraction $6 / 8=3 / 4$ can be presented as $3 / 4=1 /(1+1 / 3)$ (see Eq. (10), $k=2$ ). The truncated fraction (Eq. (11)) is $p_{1} / q_{1}=1 / 1$. The line $x+y=0$ (Eq. (13)) is shown by the dashed line. The points equivalent to $(0,0)$ on the first and second steps are shown by the arrows. Their coordinates $(13,-13)$ and $(25,-25)$ are given by equation (12) where $s=\operatorname{GCM}(6,8)=2$. The intersection point of the lines $x+y=0$ and $6 x+8 y=25 \cdot 2$ is an integer point $(25-25)$ because of $m=s=2$. The intersection point of the lines $x+y=0$ and $6 x+8 y=25$ (shown by a circle) is not integer (see the insert). 
of the $n$-th kink on the $m$-th step are

$$
x_{n, m}=\operatorname{Int}\left(n \frac{q}{p}+m \frac{r}{p}\right), \quad y_{n, m}=-n, \quad z_{n, m}=-m .
$$

This rule is also a direct mathematical corollary of the fundamental assertion. One can verify it for some $p, q, r$ (Fig. 5). The three-dimensional analogue of the Hubbard criterion can be derived from equation (8) :

The spacing between the $n$-th and the $k$-th kinks on the $m$-th $\operatorname{step}(z$ is fixed) is either Int $(|k-n| q / p)$ or Int $(|k-n| q / p)+1$. The distance in the $y$-direction between the $m$-th and the $l$-th steps (when $x$ is fixed) is either Int $(|l-m| r / q)$ or Int $(|l-m| r / q)+1$. The distance between these steps in the $x$-direction ( $y$ is fixed) is either Int $(|l-m| r / p)$ or Int $(|l-m| r / p)$ +1 .

Equation (8) yields the coordinates of all kinks on all steps. But it is clear that different steps are identical. The $m$-th step differs from the step $m=0$ only by the translation in the $x y$-plane. So, it is possible to calculate the kink coordinates $x_{n, 0}$ on the zeroth step by means of equation (8) and then obtain $x_{n, m}$ from $x_{n, 0}$ by the relation

$$
x_{n, m}=x_{n+\Delta y(m), 0}+\Delta x(m)
$$

where $(\Delta x(m), \Delta y(m))$ is a translation vector in the $x y$ plane (Fig. 5). This vector yields the coordinates of the kink on the $m$-th step which is identical to the zeroth kink on the zeroth step. The formulae for $\Delta x(m), \Delta y(m)$ are cumbersome. Prior to writing them we introduce auxiliary objects. The rational number $p / q$ must be expanded in a continued fraction :

$$
\frac{p}{q}=\frac{1}{s_{1}+\frac{1}{s_{2}+\frac{1}{\ddots}+\frac{1}{s_{k-1}+\frac{1}{s_{k}}}}}
$$

where $s_{1}, s_{2}, \ldots, s_{k}$ are positive integers. Such an expansion is well-known to be unique (see e.g. [12]). We shall call $k$ " the number of stories". Then we construct the truncated continued fraction :

$$
\frac{p_{k-1}}{q_{k-1}}=\frac{1}{s_{1}+\frac{1}{s_{2}+\frac{1}{\ddots+\frac{1}{s_{k-1}}}}}
$$

where $s_{1}, s_{2}, \ldots, s_{k-1}$ are the same as in equation (10) and $p_{k-1} / q_{k-1}$ is supposed to be irreducible. Equations (10), (11) permit $p_{k-1}$ and $q_{k-1}$ to be derived from $p, q$ unambiguously. In practice the calculation of $p_{k-1}$ and $q_{k-1}$ is trivially arithmetic (the example is given in Fig. 5). Now we consider the second analytical method which is equivalent to equation (8) :

The configuration of kinks on the $m$-th step $x_{n, m}$ is given by equation (9) where

$$
\begin{aligned}
& \Delta x(m)=(-1)^{k} \operatorname{Int}\left(\frac{r m}{s}\right) q_{k-1} \\
& \Delta y(m)=(-1)^{k+1} \operatorname{Int}\left(\frac{r m}{s}\right) p_{k-1}
\end{aligned}
$$

where $s$ is the greatest common measure of $p, q$, and $x_{n, 0}$ is given by equation (5).

Geometrically equation (12) can be treated as follows. All translation vectors $(\Delta x(m), \Delta y(m))$ lie on the straight line

$$
p_{k-1} x+q_{k-1} y=0
$$

consider the intersection point of the line (13) and the $m$-th line (7). This point may be either integer or non-integer. In the former case the point $(\Delta x(m)$, $\Delta y(m))$ is simply this intersection point. If we consider the number $m$ such that the intersection point is non-integer, then the point $(\Delta x(m), \Delta y(m))$ is an integer point belonging to the line (13). This point is below the corresponding line (7) and is the nearest to it. How to distinguish between these two cases ? The answer is trivial : the intersection point of the lines (13) and (7) is integer if and only if $s$ is a multiple of $m: m=s \cdot$ Integer, $s=\operatorname{GCM}(p, q)$.

The third analytical algorithm determining the configuration of steps and kinks also involves the translation (9), (12), but the configuration of kinks on the zeroth step $x_{n, 0}$ is defined by the recurrent procedure described in $[8,9]$. All the analytical and geometrical methods described above are quite equivalent and each of them provides entire information about the projection of the step and kink structure on the $x y$-plane. Note that these rules have been derived from the fundamental assertion by purely mathematical methods.

The second aspect of the microscopic approach is to calculate the surface tension $\alpha(\mathbf{n})$ :

$$
\alpha(\overline{\mathbf{n}})=\lim _{A \rightarrow \infty}-\frac{1}{2 A} \sum_{\overline{\mathbf{r}}} \sum_{\overline{\mathbf{r}}^{\prime}} U\left(\overline{\mathbf{r}}-\overline{\mathbf{r}}^{\prime}\right) \quad \overline{\mathbf{r}}, \overline{\mathbf{r}}^{\prime} \in \mathbb{Z}
$$

where $\overline{\mathbf{r}}$ belong to the crystal, $\overrightarrow{\mathbf{r}}$ belong to the vacuum, $A$ is the area of the interface. According to the fundamental assertion (3) the points $\overline{\mathbf{r}}$ of the crystal satisfy the inequality $\overline{\mathbf{n}} \cdot \overline{\mathbf{r}} \leqslant 0$, the vacuum points $\overline{\mathbf{r}^{\prime}}$ satisfy 
$\overline{\mathbf{n}} \cdot \overline{\mathbf{r}}^{\prime}>0$. Hence $\alpha(\overline{\mathbf{n}})$ is

$$
\begin{aligned}
\alpha(n) & =-\lim _{A \rightarrow \infty} \frac{1}{A} \frac{1}{2} \sum_{\overline{\mathbf{r}}, \overline{\mathbf{r}}^{\prime}} \theta\left(\overline{\mathbf{n}} \cdot \overline{\mathbf{r}}^{\prime}\right) \theta(-\overline{\mathbf{n}} \cdot \overline{\mathbf{r}}) U\left(\overline{\mathbf{r}}-\overline{\mathbf{r}}^{\prime}\right)= \\
& =-\lim _{A \rightarrow \infty} \frac{1}{2 A} \sum_{\overline{\mathbf{r}_{0}, \overline{\mathbf{r}}}} \theta\left(-\overline{\mathbf{n}} \cdot \overline{\mathbf{r}}_{0}\right) \theta\left(\overline{\mathbf{n}} \cdot \overline{\mathbf{r}}_{0}+\overline{\mathbf{n}} \cdot \overline{\mathbf{r}}\right) U(\overline{\mathbf{r}}) \\
& =-\frac{1}{2} \sum_{\overline{\mathbf{r}}} \theta(\overline{\mathbf{n}} \cdot \overline{\mathbf{r}}) U(\overline{\mathbf{r}})\left[\lim _{A \rightarrow \infty} \frac{1}{A} \sum_{\overline{\mathbf{r}}_{0}} \theta\left(-\overline{\mathbf{n}} \cdot \overline{\mathbf{r}}_{0}\right) \theta\left(\overline{\mathbf{n}} \cdot \overline{\mathbf{r}}_{0}+\overline{\mathbf{n}} \cdot \overline{\mathbf{r}}\right)\right] .
\end{aligned}
$$

The expression in brackets is the number of integer points locked between the parallel planes $\overline{\mathbf{n}} \cdot \overline{\mathbf{r}}_{0}=0$ and $\overline{\mathbf{n}} \cdot\left(\overline{\mathbf{r}}_{0}+\overline{\mathbf{r}}\right)=0$ (per a unit area). It is well-known that this number equals the distance $(\overline{\mathbf{n}} \cdot \mathbf{r})$ between the planes [13]. The surface tension takes the form :

$$
\alpha(n)=-\frac{1}{2} \sum_{\overline{\mathbf{r}}}(\overline{\mathbf{n}} \cdot \overline{\mathbf{r}}) \theta(\overline{\mathbf{n}} \cdot \overline{\mathbf{r}}) U(\overline{\mathbf{r}}), \quad \overline{\mathbf{r}} \in \mathbb{Z}^{3} .
$$

Note that equation (14) is an exact formula. The approximate expression for $\alpha(\overline{\mathbf{n}})$ for $U(\mathbf{r})=C / r^{\gamma}$ in the 2-D case $\overline{\mathbf{n}}=(p, q, 0)$ has been obtained previously in $[10,11]$ for simple $p / q$.

\section{Macroscopic approach.}

Now we want to calculate the shape of the whole crystal and of its facets. We do not discern the atomic structure of the facets. They become the plane regions of a finite size due to the limit in the formula (2). From the microscopic problem we shall use only the expression (14) yielding $\alpha(\overline{\mathbf{n}})$.

The ECS $\bar{R}\left(\xi_{1}, \xi_{2}\right)$ is some surface minimizing the surface energy if the volume is fixed. There is a mathematical theorem that gives the ECS if $\alpha(\bar{n})$ is known [14]. Before presenting the result we must choose two parameters $\xi_{1}, \xi_{2}$. In [14] they are two components $n_{x}, n_{y}$ of a unit normal vector $\overline{\mathbf{n}}$. The third component $n_{z}$ is their function $n_{z}=\sqrt{1-n_{x}^{2}-n_{y}^{2}}$. We retain the symmetry between $n_{x}, n_{y}, n_{z}$. The formula of [14] can be rewritten as :

Wulff theorem. The ECS $\bar{R}(\overline{\mathbf{n}})$ is

$$
\bar{R}(\overline{\mathbf{n}})=\frac{\partial \alpha}{\partial \overline{\mathbf{n}}}+\overline{\mathbf{n}}\left(\alpha(\overline{\mathbf{n}})-\overline{\mathbf{n}} \cdot \frac{\partial \alpha}{\partial \overline{\mathbf{n}}}\right)
$$

where any two independent variables determining the unit normal vector $\overline{\mathbf{n}}$ are treated as parameters of the surface $\bar{R}$, but in the derivatives all three components of $\mathbf{n}$ are considered as independent.

In other words, $\bar{R}(\overline{\mathbf{n}})$ is a function of two parameters since $|\overline{\mathbf{n}}|=1$. But the restriction $|\overline{\mathbf{n}}|=1$ must be imposed on equation (15) after differentiation. This theorem implies that the function $\alpha(\bar{n})$ is smooth. Unfortunately, as has been shown by Landau [10] the derivative $\frac{\partial \alpha}{\partial \overline{\mathbf{n}}}$ does not exist. Nevertheless, the Wulff theorem can be rederived for a particular $\alpha(\mathbf{n})$ of the form (14). The result can be symbolically written as

$$
\bar{R}(\overline{\mathbf{n}})=\frac{\partial \alpha}{\partial \overline{\mathbf{n}}}
$$

where « the derivative " $\frac{\partial \alpha}{\partial \overline{\mathbf{n}}}$ depends on the sign and on the direction of the increment $\delta \overline{\mathbf{n}}$. For example,

$\lim _{\delta n \rightarrow+0} \frac{\alpha(\overline{\mathbf{n}}+\delta n \hat{\mathbf{x}})-\alpha(\overline{\mathbf{n}})}{\delta n} \neq \lim _{\delta \boldsymbol{n} \rightarrow-0} \frac{\alpha(\overline{\mathbf{n}}+\delta n \hat{\mathbf{x}})-\alpha(\overline{\mathbf{n}})}{\delta n}$

but both limits exist. The results of this strange differentiation are given below. The explanations can be found in Appendix 2.

We start the study with the simplest two-dimensional case. Namely, consider a section of the crystal by the plane $Z=0$. The cubic symmetry implies that $n_{z}=0$. The closed curve $\bar{R}(\overline{\mathbf{n}})$ is parameterized by one variable (since the two restrictions $n_{z}=0$ and $|\overline{\mathbf{n}}|=1$ are imposed on the vector $\overline{\mathbf{n}})$. We choose $\frac{n_{x}}{n_{y}}=\operatorname{tg} \varphi=\xi$ as this parameter (Fig. 6). Mathematical properties of the contour $\bar{R}(\xi)$ appear to be rather strange.

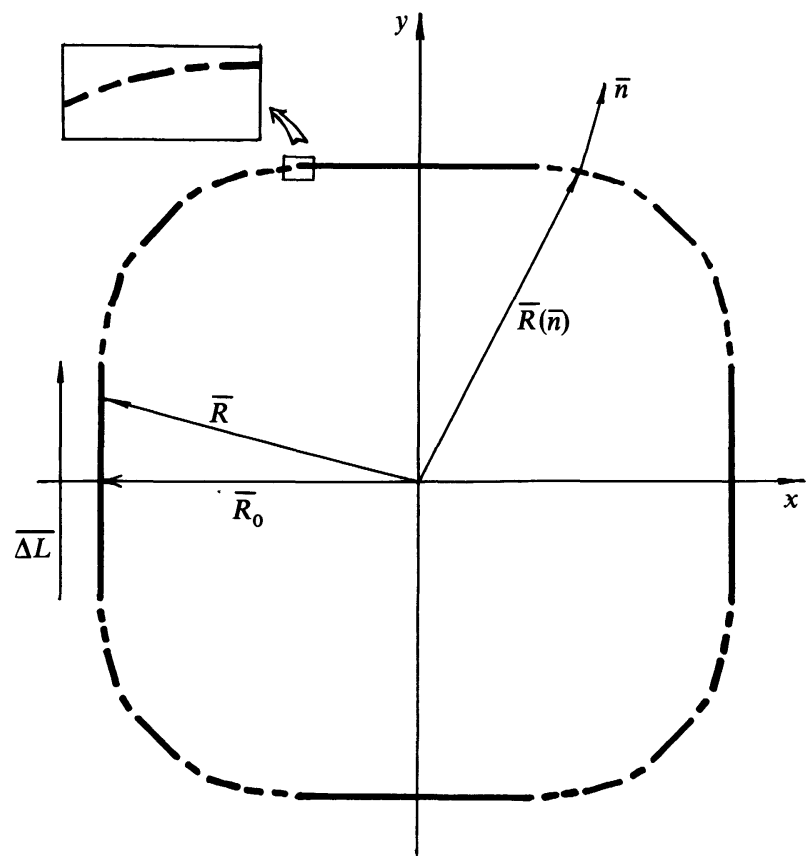

Fig. 6. - The section of the ECS by the plane $z=0$. 
Namely, the curve is convex, continuous and differentiable but only once. Moreover, the slope $\xi$ varies continuously and takes all the values $-\infty<\xi<+\infty$. It means that there is no cusp (slope discontinuity) in the curve. Nevertheless, it is completely faceted. Namely, the curve consists of an infinite number of the straight-line intervals and single points. There is no smooth rounded arc. Consider the structure of the curve more exactly. The cases of rational and irrational slopes $\xi$ must be distinguished.

First consider the former case. We fix some rational $\xi=p / q\left(\overline{\mathbf{n}}=(p, q, 0) / \sqrt{p^{2}+q^{2}}, p, q \in \mathbb{Z}\right)$. Substituting equation (14) into equation (16) we find that in the curve $\bar{R}(\xi)$ there exists an interval with the given slope $\xi=p / q$. We denote the centre of this interval by $\bar{R}_{0}(\xi)$ and the length by $\Delta L$. These are :

$$
\begin{array}{r}
\bar{R}_{0}(\overline{\mathbf{n}})=-\frac{1}{2} \sum_{\mathbf{r}} \overline{\mathbf{r}} \theta(\overline{\mathbf{n}} \cdot \overline{\mathbf{r}}) U(\mathbf{r}) \quad \\
\overline{\mathbf{r}} \in \mathbb{Z}^{3} \\
\overline{\Delta \mathbf{L}}=-\sum_{m=1}^{\infty} m \overline{\mathbf{l}} U(m \overline{\mathbf{l}}), \quad \overline{\mathbf{l}} \cdot \overline{\mathbf{n}}=0, \quad \overline{\mathbf{l}} \in \mathbb{Z}^{3}
\end{array}
$$

where $\overline{\mathbf{I}}$ is the smallest integer vector perpendicular to $\overline{\mathbf{n}}$. In this $2-\mathrm{D}$ case it is $\overline{\mathbf{I}}=(q,-p, 0)$. Thus, every rational slope $p / q$ in the curve is represented by the straight-line interval of a non-zero length $\Delta L$. The largest intervals corresponds to the basic planes $\xi=0$ and $\xi=\infty$ (Fig. 6). The intervals with large indices $p, q$ have small lengths $\Delta L$. This is because the vector $\overline{\mathbf{I}}=(q,-p, 0)$ in equation (18) increases when $p$ or $q$ rises. Remembering that $U(\overline{\mathbf{r}}) \rightarrow 0$ at $|\overline{\mathbf{r}}| \rightarrow \infty$ one can see from equation (18) that $\Delta L \rightarrow 0$ when $p, q \rightarrow \infty$. For example, if at large distances $U(\mathbf{r})=$ $C / r^{\gamma}$ then

$$
\Delta^{z} L=\frac{C \zeta(\gamma-1)}{\left(p^{2}+q^{2}\right)^{\frac{\gamma-1}{2}}} .
$$

Although the number of intervals with different rational slopes $\xi$ is infinite, the sum of their lengths is finite.

Then consider the case of an irrational slope $\xi=\frac{n_{x}}{n_{y}}$. It proves to be represented in the curve by the single point $\bar{R}_{0}(\xi)$, which is defined also by equation (17). The role of such single points is usually underestimated by physicists. The irrational slope is referred to as a mathematical abstraction. It is actually abstraction when a given irrational slope is considered. But it should be borne in mind that $\xi$ is a parameter of the curve, it takes all real values. It is well known that most of the real points are irrational (the cardinality of the set of the irrationals is continuum, but the rationals are countable). For this reason the total contribution of all the irrational slopes to some physical value may be non-zero. For example, the single points $\bar{R}_{0}(\xi)$ may contribute to the curve length. If it is so, the result of the summation of the lengths of the intervals $\Delta L(\xi)$ performed over all the rational slopes $\xi$ is smaller than the length of the whole contour. The examples of such curves are well known in mathematics. Thus the set of the single points $\bar{R}_{0}(\xi)$ with all irrational slopes $\xi=n_{x} / n_{y}$ may be important. This set is called the Cantor set. Its cardinality is continuum, but it does not contain any interval of a non-zero length. The total contribution of all the irrational slopes to the length of the curve is called the measure of the Cantor set. It may be zero or non-zero.

So the section of the ECS is a curve $\bar{R}_{0}(\bar{n})$ consisting of the straight-line intervals of the lengths $\Delta L(\mathbf{n})$ (Eq. (18)) and of the Cantor set which is determined unambiguously by equation (17). In particular, equation (17) makes it possible to calculate the measure of the Cantor set. In the framework of this lattice gas model it proves to be zero (see Appendix 1). We would like to emphasize once again that this fact is not obvious. Moreover, we conjecture that it is a consequence of the model assumption. Namely, in another model which does not suppress the atomic distortions, the measure of the Cantor set may be non-zero (compare with 1-D systems [15]). In conclusion, we mention that the so-called fractal dimension [16] of this Cantor set can also be calculated. If $U(\overline{\mathbf{r}}) \sim r^{-\gamma}$ at $|\overline{\mathbf{r}}| \rightarrow \infty$, it is equal to $2 /(\gamma-2)$.

Now consider the whole ECS in three dimensions. The surface $\bar{R}(\bar{n})$ is somewhat analogous to the above described two-dimensional curve. It is differentiable but only once and it has no cusp. The unit normal vector $\overline{\mathbf{n}}$ takes all the possible values and varies continuously. So, there is no " sharp " edge and no " sharp " corner. Nevertheless, there is no rounded area, the surface is completely faceted.

The objects of three types are presented in the ECS : they are facets, solitary edges and solitary corners. To distinguish between them one must consider some point $\bar{R}(\overline{\mathbf{n}})$ of the crystal surface. The unit vector $\overline{\mathbf{n}}$ is treated as two parameters. If they both are rational then $\bar{R}(\overline{\mathbf{n}})$ belongs to a facet. If one parameter is rational but the other is irrational then $\bar{R}(\overline{\mathbf{n}})$ belongs to a solitary edge. If the two parameters are irrational then $\bar{R}(\overline{\mathbf{n}})$ is a solitary corner. The condition of the parameters rationality must be expressed in an invariant form. Namely, the situation « two rational parameters " corresponds to $\overline{\mathbf{n}}=(p, q, r) / \sqrt{p^{2}+q^{2}+r^{2}}$. We have called this situation " two rationals " because there exist two linearly independent vectors $\overline{\mathbf{I}}_{1}$ and $\overline{\mathbf{I}}_{2}$ perpendicular to $\overline{\mathbf{n}}\left(\mathrm{e} . \mathrm{g} \cdot \overline{\mathbf{l}}_{1}=(q,-p, 0), \overline{\mathbf{I}}_{2}=(r, 0,-p)\right.$, $p, q, r \in \mathbb{Z})$. If only one integer vector $\overline{\mathbf{I}}$ perpendicular to $\overline{\mathbf{n}}$ exists we call the situation « one rational, one irrational ». If there is no such vector, then two parameters are irrational. Remembering that the unit vector $\overline{\mathbf{n}}$ perpendicular to the crystal surface is regarded as two parameters, we present the formulae determining the ECS $\bar{R}(\overline{\mathbf{n}})$.

For any $\overline{\mathbf{n}}$ such that there is no integer vector perpendicular to $\overline{\mathbf{n}}$ there is only one point $\bar{R}_{0}(\overline{\mathbf{n}})$ in the 
ECS. It is called a solitary corner, $\bar{R}_{0}(\overline{\mathbf{n}})$ is given by equation (17).

For any $\overline{\mathbf{n}}$ such that there exists only one integer vector $\overline{\mathbf{I}}=\left(l_{x}, l_{y}, l_{z}\right)$ perpendicular to $\overline{\mathbf{n}}$

$$
\begin{array}{cc}
\overline{\mathbf{I}} \cdot \overline{\mathbf{n}}=0 \quad \text { or } & l_{x} n_{x}+l_{y} n_{y}+l_{z} n_{z}=0, \\
& l_{x}, l_{y}, l_{z} \in \mathbb{Z}
\end{array}
$$

there is a straight-line interval in the ECS called a solitary edge

$$
\bar{R}(\overline{\mathbf{n}})=\bar{R}_{0}(\overline{\mathbf{n}})+t \overline{\Delta L}(\overline{\mathbf{n}}), \quad-\frac{1}{2} \leqslant t \leqslant+\frac{1}{2} .
$$

It is parallel to $\overline{\mathbf{l}}$, its length $\overline{\Delta \mathbf{L}}$ is given by equation (18), its centre is in the point $\bar{R}_{0}(\overline{\mathbf{n}})$ given by equation (17).

Before presenting the results for the situation " two rationals " we need some definitions. Denote the centre of the facet by $\bar{R}_{0}(\overline{\mathbf{n}})$. The facet boundary is denoted by $\bar{R}_{0}(\overline{\mathbf{n}})+\overline{\mathbf{\rho}}(\overline{\mathbf{n}}, \overline{\mathbf{e}})$, the vector $\overline{\mathbf{\rho}}(\overline{\mathbf{n}}, \overline{\mathbf{e}})$ belonging to the facet : $\overline{\boldsymbol{\rho}}(\overline{\mathbf{n}}, \overline{\mathbf{e}}) \cdot \overline{\mathbf{n}}=0$ (Fig. 7). The vector $\overline{\mathbf{e}}$ is a unit vector perpendicular to the contour $\overline{\boldsymbol{\rho}}(\overline{\mathbf{n}}, \mathbf{e})$ and lying in the plane of the facet : $\overline{\mathbf{e}} \cdot \overline{\mathbf{n}}=0$. The designation $\overline{\boldsymbol{\rho}}(\overline{\mathbf{n}}, \overline{\mathbf{e}})$ implies that the facet boundary is parametrized by the unit normal $\overline{\mathbf{e}}(\overline{\mathbf{e}}$ is one parameter because of the two restrictions $\overline{\mathbf{e}} \cdot \overline{\mathbf{n}}=0,|\overline{\mathbf{e}}|=1$ ). Such parameterization is useful because the contour $\overline{\boldsymbol{\rho}}(\overline{\mathbf{n}}, \overline{\mathbf{e}})$ will prove to resemble the section of the ECS described above. It consists of an infinite number of edges and of the Cantor set. The parameter $\overline{\mathbf{e}}$ varies continuously and takes all possible values. If it is rational the edge of the length $\Delta L$ appears in the contour. The centre of the edge is denoted as $\overline{\boldsymbol{\rho}}_{0}(\overline{\mathbf{n}}, \overline{\mathbf{e}})$. If the parameter $\overline{\mathbf{e}}$ is irrational there is a unique point $\overline{\boldsymbol{\rho}}_{0}(\overline{\mathbf{n}}, \overline{\mathbf{e}})$ in the contour. The only difference between the curve in figure 6 and the facet boundary $\overline{\boldsymbol{\rho}}(\overline{\mathbf{n}}, \mathbf{e})$ (Fig. 7) is the absence of the cubic symmetry in the latter curve. The formulae defining $\overline{\boldsymbol{\rho}}(\overline{\mathbf{n}}, \mathbf{e})$ (see below) contain the sum over the "Diophantine" points, i.e. the integer points lying in the plane $\overline{\mathbf{r}} \cdot \overline{\mathbf{n}}=0, \overline{\mathbf{r}} \in \mathbb{Z}^{3}$. They form a simple two-dimensional lattice (see Fig. 3) with the basic vectors $\overline{\mathbf{a}}_{1}, \overline{\mathbf{a}}_{2}$ which are well known in the theory of the Diophantine equations [13]. (Note that the condition $\overline{\mathbf{n}} \cdot \overline{\mathbf{r}}=0, \overline{\mathbf{r}} \in \mathbb{Z}^{3}$ can be written as the Diophantine equation $p x+q y+r z=0, p, q, r$; $x, y, z \in \mathbb{Z}$.) The vectors $\overline{\mathbf{a}}_{1}, \overline{\mathbf{a}}_{2}$ are expressed by means of the continued fraction expansions of $p / q, p / r$ or $q / r$. We denote the greatest common measure (GCM) of $p$ and $q$ as $s$ and analogously :

$$
\begin{array}{lll}
s=\operatorname{GCM}(p, q) & t=\operatorname{GCM}(p, r) & l=\operatorname{GCM}(q, r) \\
p=s t \tilde{p} & q=s l \widetilde{q} & r=t l \tilde{r} .
\end{array}
$$

The ratios $\tilde{p} / \tilde{q}, \tilde{p} / \tilde{r}, \tilde{q} / \tilde{r}$ are irreducible. Then we must expand $\tilde{p} / \tilde{q}$ in a continued fraction analogous to equation (10) and calculate a truncated one $\tilde{p}_{k-1} / \tilde{q}_{k-1}$ as in equation (11). Now we give the general result. The explanations can be found in Appendix 2.

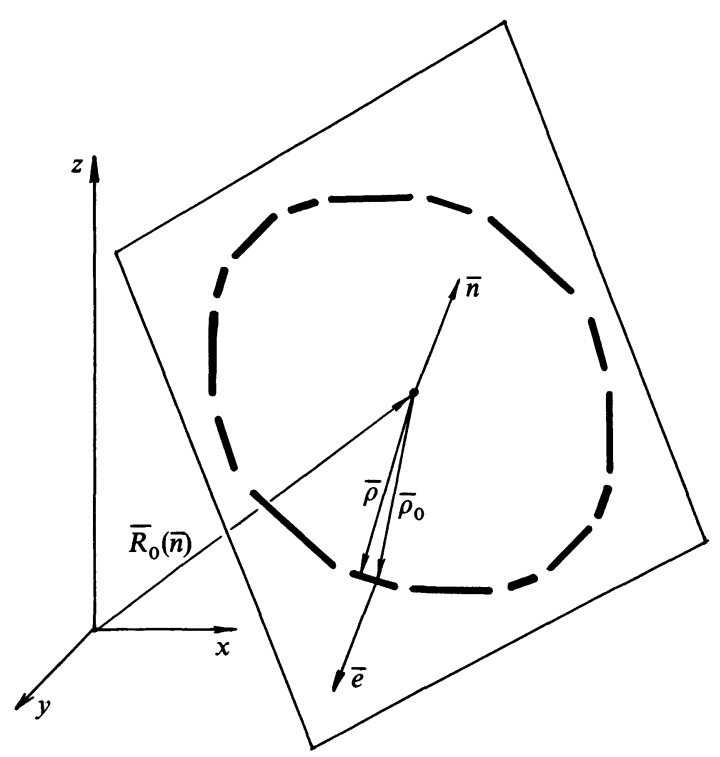

Fig. 7. - The boundary of a facet with a normal $\overline{\mathbf{n}}$. The plane shown in the figure is given by Wulff's theorem : $\overline{\mathbf{R}} \cdot \overline{\mathbf{n}}=\alpha(\overline{\mathbf{n}})$.

For $\overline{\mathbf{n}}=(p, q, r) / \sqrt{p^{2}+q^{2}+r^{2}}, p, q, r \in \mathbb{Z}$ there is a facet in the ECS

$$
\bar{R}(\overline{\mathbf{n}})=\bar{R}_{0}(\overline{\mathbf{n}})+t \overline{\mathbf{p}}(\overline{\mathbf{n}}, \overline{\mathbf{e}}) \quad 0 \leqslant t \leqslant 1 .
$$

Its centre $\bar{R}_{0}(\overline{\mathbf{n}})$ is given by equation (17). The facet boundary

$$
\bar{R}_{0}(\overline{\mathbf{n}})+\overline{\mathbf{\rho}}(\overline{\mathbf{n}}, \overline{\mathbf{e}})
$$

is determined by the following formulae (the unit vector $\overline{\mathbf{e}}$ normal to the contour is regarded as a parameter). For $\overline{\mathbf{e}}$ such that there is no integer vector perpendicular to both $\overline{\mathbf{e}}$ and $\overline{\mathbf{n}}$ there is only one point

$$
\overline{\mathbf{\rho}}(\overline{\mathbf{n}}, \overline{\mathbf{e}})=\overline{\boldsymbol{\rho}}_{0}(\overline{\mathbf{n}}, \overline{\mathbf{e}})
$$

determined by

$$
\overline{\boldsymbol{\rho}}_{0}(\overline{\mathbf{n}}, \overline{\mathbf{e}})=-\frac{1}{2} \sum_{\mathbf{r}=m \mathbf{a}_{1}+j \mathbf{a}_{2}} \overline{\mathbf{r}} \theta(\overline{\mathbf{e}} \cdot \overline{\mathbf{r}}) U(\overline{\mathbf{r}})
$$

where the summation is performed over the 2-D lattice of the "Diophantine " points $\left(\overline{\mathbf{r}} \cdot \overline{\mathbf{n}}=0, \overline{\mathbf{r}} \in \mathbb{Z}^{3}\right)$. The basic vectors of this lattice are

$$
\begin{aligned}
& \overline{\mathbf{a}}_{1}=(l \widetilde{q},-t \widetilde{p}, 0) \\
& \overline{\mathbf{a}}_{2}=\left(l \tilde{r} \widetilde{q}_{k-1},-t \tilde{p} \widetilde{p}_{k-1},(-1)^{k} s\right)
\end{aligned}
$$

$s, l, t, \tilde{p}, \tilde{q}, \tilde{r}$ are defined in equation (20), $k$ is the number of the stories of the continued fraction $\tilde{p} / \tilde{q}$. For $\overline{\mathbf{e}}$ such that there is an integer vector $\overline{\mathbf{l}}$ perpendicular to both $\overline{\mathbf{e}}$ and $\overline{\mathbf{n}}$ there is a straight-line interval (an edge) in the 
contour

$$
\bar{\rho}(\overline{\mathbf{n}}, \overline{\mathbf{e}})=\overline{\boldsymbol{\rho}}_{0}(\overline{\mathbf{n}}, \overline{\mathbf{e}})+t \overline{\Delta \mathbf{L}} \quad-\frac{1}{2} \leqslant t \leqslant \frac{1}{2} .
$$

Its centre is in the point $\overline{\boldsymbol{\rho}}_{0}(\overline{\mathbf{n}}, \overline{\mathbf{e}})$ given by equation (21). The smooth edge is parallel to $\bar{I}$ and is of length $\Delta L$ given by equation (17).

The ECS $\bar{R}(\overline{\mathbf{n}})$ is shown schematically in figure 8 . The facets $\{001\},\{100\},\{010\}$ are the largest. Their boundaries resemble the section of the ECS by the plane $Z=0$ (Fig. 6). Other facets are smaller and asymmetric (Fig. 7). It should be noted that the length of the smooth edge $\Delta L(\bar{n}, \bar{e})$ is given by equation (18) and depends only on the orientation of the edge $\overline{\mathbf{l}}$. It is independent of the facet indices. Therefore, parallel edges belonging to different facets have the same length. This property clarifies the appearance of solitary edges, which also have the same length (18). We consider the simplest example $n_{z}=0$. There is an infinite number of facets $\{p, q, 0\}$. In the boundary of each facet of this type there are two edges with the orientation $\overline{\mathbf{l}}=(0,0,1)$ (vertical intervals in Fig. 8). The lengths of these edges are $p, q$-independent. On the other hand, the facet $\{p, q, 0\}$ shrinks when $p, q$ tend to infinity. It is clear from equations (18), (22) that the facet size in the $x y$-direction tends to zero when $p, q \rightarrow \infty$, but the size in the $Z$-direction remains constant (Fig. 8). Hence, vertical solitary edges are created by the shrunk facets $\{p, q, 0\}$. Solitary edges of an arbitrary orientation $\overline{\mathbf{I}}$ are created similarly.

Solitary corners and solitary edges in the ECS form the Cantor sets. In the framework of this model their measures are zero. It means that the area of the surface is equal to the sum of the areas of all facets. Analogously, the length of the boundary of each facet equals the sum of all edges.

\section{Concluding remarks.}

We conjecture that the sets of solitary edges and corners may have a non-zero measure when atomic distortions are considered. This assumption is based on the analogy with 1-D systems. Aubry [15] has shown that in a discrete elastic chain the corresponding Cantor set has a non-zero measure. It should be emphasized that Aubry's theory describes various real physical systems. So the Cantor set of the non-zero measure is not a mathematical abstraction. It can be observed experimentally [15].

The role of quantum and thermal fluctuations is not clear. According to modern theories $[3,4,11]$ the temperature suppresses facets with large indices. The critical temperature depends on $p, q, r$ and tends to zero when $p, q, r \rightarrow \infty$. At finite temperature the ECS consists of a finite number of facets with small indices and of smoothed regions. The latter are changed by the new facets with larger indices as $T \rightarrow 0$. This mechanism of creating the "Devil's staircase structure " has been proposed in $[11,19]$. It is noteworthy

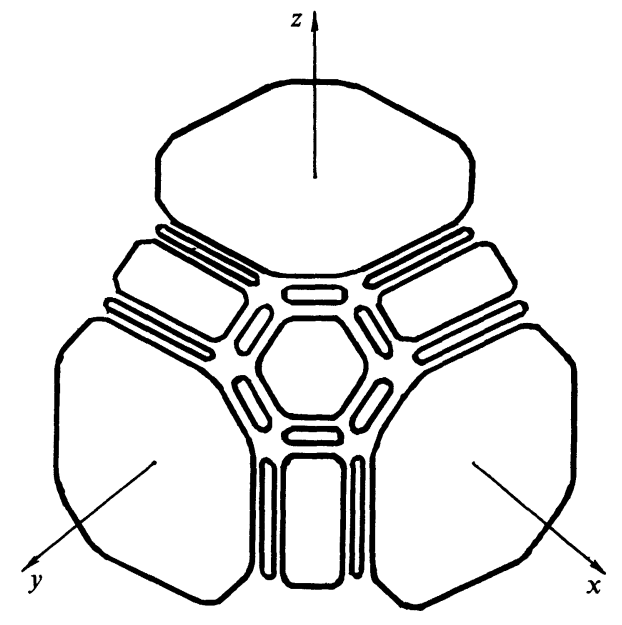

Fig. 8. - The equilibrium crystal shape.

that finite temperature theories are rather approximate. The exact calculations has not been performed (the only exception is the work [19], but, unfortunately, the model does not allow to investigate high index facets). As it has been mentioned above, fluctuations in experimentally studied samples of both ${ }^{4} \mathrm{He}$ and $\mathrm{Pb}$ are important. For this reason the measured ECS differ from the calculated zero-temperature ECS. Nevertheless, the ECS of both $\mathrm{Pb}$ [17] and solid ${ }^{4} \mathrm{He}$ [18] exhibit smooth edges. The Cantor set is destroyed by fluctuations or has not been discerned. In any event, the slope is continuous.

In conclusion, it should be mentioned that our theory describes also two-dimensional systems. In this case the cubic lattice must be replaced by a quadratic lattice. The ECS is the contour shown in figure 6. It is given analytically by equations (17), (18) where $\mathbb{Z}^{3}$ must be substituted by $\mathbb{Z}^{2}$. The solution of the microscopic problem is given by equation (5). In particular the ECS of a 2-D crystal with regular triangular lattice can be easily calculated by means of equations (17) and (18). The contour appears to have the $\mathrm{C}_{6}$-symmetry. The lattice gas model is quite adequate for 2-D crystals. Namely, consider a monolayer of atoms adsorbed on a crystal substrate. The periodic substrate potential is often much stronger than the interaction $U(\mathbf{r})$ between the adsorbed atoms. In this case the atoms can occupy only the minima of the potential. Such a system is described by the lattice gas model.

\section{Acknowledgments.}

The author thanks V. L. Pokrovsky for useful discussions.

\section{Appendix 1.}

2-D CRYSTAL, MICROSCOPIC PROBLEM. - There is an exact analogy between the 1-D lattice gas with an infinite range of interaction [6-8] and the 2-D version of 
the model considered above. A step on the crystal boundary is treated as a particle. To know the structure of the interface is to know the configuration of the steps $\left\{x_{n}\right\}$. The energy of step creation is :

$$
\varepsilon=-\frac{1}{2} \sum_{m=1}^{\infty} m U(m \hat{\mathbf{x}})
$$

where $\hat{\mathbf{x}}, \hat{\mathbf{y}}$ are unit vectors parallel to the $x$ and $y$ axes, we consider the case $p \leqslant q$. The interaction between the $n$-th and the $(n+m)$-th steps is

$J_{m}\left(x_{n+m}-x_{n}\right)=-\sum_{k=1}^{\infty} k U\left(m \hat{\mathbf{y}}+\left(x_{n+m}-x_{n}+k\right) \hat{\mathbf{x}}\right)$

where $x_{n+m}-x_{n} \geqslant 0, m \geqslant 1$. Note that the interaction depends on the step spacing and on the number $m$ of other steps located between them. The case $x_{n+m}-$ $x_{n}=0$ describes one step of the height $m$. The slope $p / q$ serves as the step concentration. If it is fixed, the coordinates of 1-D lattice gas particles are given by the Aubry formula $x_{n}=\operatorname{Int}(n q / p)$ [6] which coincides with equation (5). The configuration $\left\{x_{n}\right\}$ of particles (steps) can also be obtained by the recurrent procedure described in $[8,9]$. It is known in 1-D theory [6-9] that the interaction $J(x)$ must be a convex function of $x$ :

$$
\mathfrak{J}_{m}(x+1)-2 \mathfrak{J}_{m}(x)+\mathfrak{J}_{m}(x-1)>0 .
$$

Substituting equation (24) into (25) we find that the 1.h.s. of equation (25) reduces to $-U(x \hat{\mathbf{x}}+m \hat{\mathbf{y}})$ which is positive by definition.

\section{Appendix 2.}

THE FACET BOUNDARY. - Consider a facet $\{p, q, r\}$. Its boundary can be symbolically written in the form of (16). The derivative (16) denotes a set of the limits

$$
\lim _{|\delta \overline{\mathbf{n}}| \rightarrow 0} \frac{\alpha(\overline{\mathbf{n}}+\delta \overline{\mathbf{n}})-\alpha(\mathbf{n})}{|\delta \overline{\mathbf{n}}|} \frac{\delta \overline{\mathbf{n}}}{|\delta \overline{\mathbf{n}}|}
$$

which depend on the $\delta \overline{\mathbf{n}}$ direction. If some $\delta \overline{\mathbf{n}} /|\delta \overline{\mathbf{n}}|$ is fixed it is evident that the corresponding limit (26) determines the radius-vector of the facet boundary. The direction of this radius-vector coincides with $\delta \overline{\mathbf{n}}$. Substituting (14) into (26) we find that there is a regular part in (26). It is independent of $\delta \overline{\mathbf{n}}$ and is equal to (17). The "strange part " of (26) appears when $\delta \bar{n}$ is perpendicular to $\overline{\mathbf{n}}$. It yields the vector $\overline{\boldsymbol{\rho}}(\overline{\mathbf{n}}, \overline{\mathbf{e}})$ belonging to the facet (Fig. 7). The change of $\overline{\mathbf{n}}$ such that $\delta \overline{\mathbf{n}} \cdot \overline{\mathbf{n}}=0$ means that the plane of the facet is rotated by a small angle $|\delta \overline{\mathbf{n}}|$. The step function $\theta(\overline{\mathbf{n}} \cdot \overline{\mathbf{r}})$ in equation (14) has a singularity in $\overline{\mathbf{n}} \cdot \overline{\mathbf{r}}=0, \overline{\mathbf{r}} \in \mathbb{Z}^{3}$, i.e. in the « Diophantine " points. For this reason only these points contribute to the "strange part". Calculating the limit (26) we find that « the strange part » is equal to

$$
\overline{\mathbf{\rho}}(\overline{\mathbf{n}}, \overline{\mathbf{e}})=\frac{\partial \widetilde{\alpha}(\overline{\mathbf{n}}, \overline{\mathbf{e}})}{\partial \overline{\mathbf{e}}}
$$

where $\alpha(\overline{\mathbf{e}})$ is the surface tension of the 2-D lattice of the «Diophantine" points :

$$
\alpha(\overline{\mathbf{n}}, \overline{\mathbf{e}})=-\frac{1}{2} \sum_{\overline{\mathbf{r}}=m \overline{\mathbf{a}}_{1}+j \overline{\mathbf{a}}_{2}}(\overline{\mathbf{e}} \cdot \overline{\mathbf{r}}) \theta(\overline{\mathbf{e}} \cdot \overline{\mathbf{r}}) U(\overline{\mathbf{r}}) .
$$

Thus, the problem of the facet boundary is reduced to the problem of finding the ECS of a 2-D crystal. The "Diophantine" lattice is not quadratic, so $\overline{\boldsymbol{\rho}}(\mathbf{e})$ is asymmetric. Nevertheless, the solution is of the form (22), (18). Note, that the 2-D microscopic problem can be also solved. The coordinates of steps on an edge with the orientation $\overline{\mathbf{e}}=p \overline{\mathbf{a}}_{1}+q \overline{\mathbf{a}}_{2}$ are given by equation (5), where $x_{n}$ and $y_{n}$ are the projections on $\overline{\mathbf{a}}_{1}$ and $\overline{\mathbf{a}}_{2}$.

\section{Appendix 3.}

ProOf OF THE Fundamental assertion. - Consider a plane with fixed normal $\overline{\mathbf{n}}$. This plane is given by the equation $\overline{\mathbf{n}} \cdot \overline{\mathbf{r}}=0$. In coordinates it is written as

$$
n_{x} x+n_{y} y+n_{z} z=0
$$

$\left(n_{x}, n_{y}, n_{z}\right.$ are not necessarily integer). We denote a set of integer points lying below this plane and on the plane as $Z_{-}$. A set of points lying above the plane (29) will be called $\mathrm{Z}_{+}$:

$$
Z_{-}=\left\{\overline{\mathbf{r}} \in \mathbb{Z}^{3} \mid \overline{\mathbf{n}} \cdot \overline{\mathbf{r}} \leqslant 0\right\}, \quad Z_{+}=\left\{\overline{\mathbf{r}} \in \mathbb{Z}^{3} \mid \overline{\mathbf{n}} \cdot \overline{\mathbf{r}}>0\right\} .
$$

The configuration of atoms such that $Z_{-}$is occupied by atoms and $Z_{+}$is empty will be called a unreconstructed configuration (Figs. 3a, 4). We must consider any other configuration with the same average slope (see e.g. Fig. 2). More exactly, we consider only finite perturbations of the unreconstructed configuration : the reconstructed configuration is obtained from the unreconstructed one by occupying a finite number of points which were empty and by evacuating a finite number of atoms from $Z_{-}$. The total number of atoms must not be conserved. The energy of each configuration is given by the formal sum :

$$
\boldsymbol{E}=-\sum_{\mathbf{r}, \overline{\mathbf{r}}^{\prime}} U\left(\overline{\mathbf{r}}-\overline{\mathbf{r}^{\prime}}\right) \quad \overline{\mathbf{r}} \in \mathbf{Z}_{+}, \overline{\mathbf{r}}^{\prime} \in \mathbf{Z}_{-} .
$$

A correct mathematical object is the difference in energy for two configurations, it is finite (compare with [15]).

The following theorem applies :

For any given orientation $\overline{\mathbf{n}}$ the unreconstructed configuration is a ground state configuration in the sense that the difference in energies (30) between any reconstructed configuration and the unreconstructed one is positive.

Below is given the Kerimov's proof : In the unreconstructed configuration $Z_{-}$is occupied, $Z_{+}$is empty. Consider the simplest finite perturbation : some point $\overline{\mathbf{r}}_{0} \in Z_{+}$is occupied by an atom. The energy difference is

$$
\Delta \varepsilon=\sum_{\overline{\mathbf{r}} \in \mathbf{Z}_{-}} U\left(\overline{\mathbf{r}}-\overline{\mathbf{r}}_{0}\right)-\sum_{\overline{\mathbf{r}} \in \mathbf{Z}_{+}} U\left(\overline{\mathbf{r}}-\overline{\mathbf{r}}_{\mathbf{0}}\right) .
$$


For any $\overline{\mathbf{r}} \in Z_{\text {- denote }} \bar{R}(\mathbf{r})=2 \overline{\mathbf{r}}_{0}-\overline{\mathbf{r}}$. The point $\bar{R}(\overline{\mathbf{r}})$ belongs to $Z_{+}$. Really, $\overline{\mathbf{n}} \cdot \bar{R}(\overline{\mathbf{r}})=2 \overline{\mathbf{n}} \cdot \overline{\mathbf{r}}_{0}-\overline{\mathbf{n}} \cdot \overline{\mathbf{r}}$; $\overline{\mathbf{n}} \cdot \overline{\mathbf{r}} \leqslant 0$ since $\overline{\mathbf{r}}_{0} \in Z_{+} ; \overline{\mathbf{n}} \cdot \overline{\mathbf{r}}_{0}>0$ since $\overline{\mathbf{r}}_{0} \in \mathbf{Z}_{+} ;$thus

$$
\overline{\mathbf{n}} \cdot \bar{R}(\overline{\mathbf{r}}) \geqslant 2 \overline{\mathbf{n}} \cdot \overline{\mathbf{r}}_{0}>0
$$

it means that for any $\overline{\mathbf{r}} \in Z_{\text {- }}$ the point $\bar{R}(\mathbf{r}) \in Z_{+}$. Note that due to $\overline{\mathbf{r}}-\overline{\mathbf{r}}_{0}=\overline{\mathbf{r}}_{0}-\bar{R}(\overline{\mathbf{r}})$ and to $U(\overline{\mathbf{r}})=U(-\overline{\mathbf{r}})$ we have $U\left(\overline{\mathbf{r}}-\overline{\mathbf{r}}_{0}\right)=U\left(\bar{R}(\overline{\mathbf{r}})-\overline{\mathbf{r}}_{0}\right)$. Thus every addend $U\left(\overline{\mathbf{r}}-\overline{\mathbf{r}}_{0}\right)$ in the first sum in (31) is cancelled by the corresponding addendum $U\left(\bar{R}(\overline{\mathbf{r}})-\overline{\mathbf{r}}_{0}\right)$ in the second sum. There is an infinite number of points $\overline{\mathbf{r}} \in Z_{+}$such that they have no corresponding points in $Z_{-}$. Really, for any $\overline{\mathbf{r}} \in Z_{-}$the point $\bar{R}(\mathbf{r})$ lies below the plane $\overline{\mathbf{n}} \cdot\left(\bar{R}(\overline{\mathbf{r}})-2 \overline{\mathbf{r}}_{0}\right)=0$, which is parallel to (29). The points between these two parallel planes have no corresponding points in $Z_{-}$. Therefore (31) becomes

$$
\begin{array}{r}
\Delta \mathcal{E}=-\sum U\left(\overline{\mathbf{r}}-\overline{\mathbf{r}}_{0}\right) \theta(\overline{\mathbf{n}} \cdot \overline{\mathbf{r}}) \theta\left[-\overline{\mathbf{n}} \cdot\left(\overline{\mathbf{r}}-2 \overline{\mathbf{r}}_{0}\right)\right], \\
\overline{\mathbf{r}} \in \mathbb{Z}^{3}
\end{array}
$$

which is positive due to $U(\mathbf{r})<0$.
The case of the evacuating of one atom from $Z_{-}$is treated analogously. Now consider an arbitrary finite perturbation. The points $\overline{\mathbf{r}}_{1}, \overline{\mathbf{r}}_{2}, \ldots, \overline{\mathbf{r}}_{k} \in Z_{+}$are occupied, $\overline{\mathbf{r}}_{k+1}, \ldots, \overline{\mathbf{r}}_{n} \in Z_{-}$are empty. The energy difference is

$$
\begin{aligned}
\Delta \mathcal{E}= & \sum_{i=1}^{k}\left[\sum_{\overline{\mathbf{r}} \in \mathbf{Z}_{-}} U\left(\overline{\mathbf{r}}-\overline{\mathbf{r}}_{i}\right)-\sum_{\overline{\mathbf{r}} \in \mathbf{Z}_{+}} U\left(\overline{\mathbf{r}}-\overline{\mathbf{r}}_{i}\right)\right]+ \\
& +\sum_{i=k}^{n}\left[\sum_{\overline{\mathbf{r}} \in \mathbf{Z}_{+}} U\left(\overline{\mathbf{r}}-\overline{\mathbf{r}}_{i}\right)-\sum_{\overline{\mathbf{r}} \in \mathbf{Z}_{-}} U\left(\overline{\mathbf{r}}-\overline{\mathbf{r}}_{i}\right)\right] .
\end{aligned}
$$

Fixing some $\overline{\mathbf{r}}_{i} \in \mathrm{Z}_{+}$we have the sum analogous to (31). For any $\overline{\mathbf{r}} \in Z_{-}$we put $\bar{R}_{i}(\overline{\mathbf{r}})=2 \overline{\mathbf{r}}_{i}-\overline{\mathbf{r}}$ if this point is empty in the new configuration and $\bar{R}_{i}(\mathbf{r})=$ $\left(2 \overline{\mathbf{r}}_{i}-\overline{\mathbf{r}}\right)+\left(\overline{\mathbf{r}}_{i}-\overline{\mathbf{r}}\right)$ in the other case. If this point is also occupied we try to put $\bar{R}_{i}(\mathbf{r})=4 \overline{\mathbf{r}}_{i}-3 \overline{\mathbf{r}}$, $\bar{R}_{i}=5 \overline{\mathbf{r}}_{i}-4 \overline{\mathbf{r}}$, etc. until the point $(m+1) \overline{\mathbf{r}}_{i}-m \overline{\mathbf{r}}$ is empty. Analogously for any $\overline{\mathbf{r}} \in Z_{-}$we find $\bar{R}_{k+1}(\mathbf{r})$, $\bar{R}_{k+2}(\mathbf{r}), \ldots, \bar{R}_{n}(\mathbf{r})$. It can be easily seen that every negative term $U\left(\overline{\mathbf{r}}-\overline{\mathbf{r}}_{i}\right)$ in (34) is cancelled by the corresponding term $U\left(\bar{R}_{i}(\overline{\mathbf{r}})-\overline{\mathbf{r}}_{i}\right)$. An infinite number of positive terms remains uncancelled. Therefore, the unreconstructed configuration is a ground state.

\section{References}

[1] Keshishev, K. O., Parshin, A. Ya., Babkin, A. V., Sov. Phys. JETP 53 (1981) 362. For a review see :

Keshishev, K. O., Parshin, A. Ya., Shalnikov, A. I. in Soviet Sci. Reviews, I. M. Khalatnikov, ed. (Harwood Academic, Chur) 1982, vol. 4, p. 155.

[2] Wolf, P. E., Balibar, S., Gallet, F., Phys. Rev. Lett. 51 (1983) 1366.

[3] Fisher, D. S., WeEks, J. D., Phys. Rev. Lett. 50 (1983) 1077.

[4] Iordansky, S. V., Korshunov, S. E., Sov. Phys., JETP Lett. 38 (1983) 542. JETP Lett. 39 (1984) 466.

[5] Kerimov, A., Private communication (1984).

[6] Aubry, S., J. Phys. C 16 (1983) 2497.

[7] Habbard, J., Phys. Rev. B 17 (1978) 494.

[8] Burkov, S. E., SinaI, Ya. G., Soviet Math. Surveys 38 (1983) 205.

Burkov, S. E., J. Physique Lett. 44 (1983) L-179.

[9] BuRKov, S. E., Fractally charged discommensurations in a discrete chain, to be published.
[10] LANDAU, L. D. in the Anthology celebrating the 70th anniversary of A. F. Ioffe (USSR Academy of Sciences, Moscow) 1950, p. 44 (Russian). See also Collected works by L. D. Landau translated by D. Ter-Haar (Pergamon, London) 1971.

[11] Bolshov, L. A., Pokrovsky, V. L., Uimin, G. V., Sov. Phys., JETP Lett. 39 (1984) 145.

[12] Khintchin, A. Ya., Continued Fractions (Dover Publ.) 1967.

[13] Vinogradov, I. M., Foundations of Number Theory (Nauka, Moscow, Russian), 1972.

[14] WulfF, G., Z. Kristallogr. Mineral. 34 (1901) 449, see also : L. D. Landau, E. M. Lifshitz, Statistical Physics (Pergamon, London) 1962, § 155.

[15] Aubry, S., J. Physique 44 (1983) 147.

[16] Mandelbrot, B., The Fractal Geometry of Nature (Freeman, San-Francisco) 1982.

[17] Rottman, C., Wortis, M., Heyraud, J. C., Metois, J. M., Phys. Rev. Lett. 52 (1984) 1009.

[18] BabKin, A. V., Keshishev, K. O., KoPeliovitCh, D. B., PARShin, A. Ya., Sov. Phys., JETP Lett. 39 (1984) 519.

[19] Rottman, C., Wortis, M., Phys. Rep. 103 (1984) 59. 